\title{
EFEITO DA DOSE E EXPOSIÇÃO À TERRA DE DIATOMÁCEA DE DIFERENTES INSETOS EM MILHO ARMAZENADO
}

\author{
L.E.G. Antunes, E. Ferrari Filho, R. Gottardi, J. Sant'Ana, R.G. Dionello
}

Universidade Federal do Rio Grande do Sul, Departamento de Fitossanidade, Av. Bento Gonçalves, 7712, CEP 91540-000, Porto Alegre, RS, Brasil. E-mail: luidieric.antunes@gmail.com

\section{RESUMO}

Sitophilus zeamais e Tribolium castaneum são importantes insetos-pragas de grãos armazenados e seus danos correspondem a aproximadamente $25 \%$ das perdas na pós-colheita do Brasil. Entre as formas de controle destaca-se o uso de pós-inertes. Sendo assim, o objetivo deste trabalho foi avaliar a mortalidade de adultos de $S$. zeamais e $T$. castaneum com uso de terra de diatomácea (TD) em diferentes dosagens e períodos de exposição. Os tratamentos com terra de diatomácea foram: 1.000 e 2.000 g.t t $^{-1}$ e controle (sem aplicação). Os insetos (10 de cada espécie) foram acondicionados em recipientes plásticos de $300 \mathrm{~mL}$, contendo $100 \mathrm{~g}$ de grãos de milho com diferentes umidades (12, 14 e 16\% b.u.). As infestações ocorreram 1 hora, 10 e 20 dias após a aplicação da TD. Avaliou-se a mortalidade aos 30 e 60 dias. Foram realizadas cinco repetições/tratamento. Obteve-se maior mortalidade para a espécie S. zeamais do que T. castaneum, sendo que ela aumenta conforme o tempo de exposição dos insetos. Conclui-se que a espécie $T$. castaneum apresenta maior tolerância e que quanto maior tempo os insetos estiverem em contato com grãos tratados maior será a mortalidade.

PALAVRAS-CHAVE: Sitophilus zeamais, Tribolium castaneum, pó-inerte.

\section{ABSTRACT}

EFFECT OF DOSE AND EXPOSURETODIATOMACEOUSEARTHONDIFFERENT INSECTS IN STORED CORN. Sitophilus zeamais and Tribolium castaneum are important insect pests of stored grain and their damage corresponds to approximately $25 \%$ of post-harvest losses in Brazil. The methods used for the control of these pests notably include the use of post-harvest inert dusts. Therefore, the objective was to assess the mortality of adult $S$. zeamais and T. castaneum with the use of diatomaceous earth (DE) at different dosages and durations. The treatments with diatomaceous earth were 1,000 and 2,000 GT-1 and control (no application). Insects (10 each species) were placed in 300-ml plastic containers containing $100 \mathrm{~g}$ of corn kernels with different moisture contents (12, 14 and 16\% wb). Infestations occurred 1 hour, 10 and 20 days after the application of TD. Mortality was evaluated at 30 and 60 days. There were five replicates per treatment. There was a higher mortality for the species $S$. zeamais than for T. castaneum, and this morality rate increased in direct relation to insect exposure time. It was concluded that T. castaneum has greater tolerance and that the more time the insects are in contact with treated grain the greater the mortality.

KEY WORDS: Sitophilus zeamais, Tribolium castaneum, inert powder.

\section{INTRODUÇÃO}

Estima-se que, no Brasil, as perdas nas etapas de pós-colheita de grãos estejam entre 25 e $30 \%$ do queé produzido, sendo estas causadas principalmente por insetos-pragas, fungos e roedores (LoRINI et al., 2002).

Um dos entraves na armazenagem do milho está relacionado à presença de insetos pragas. Dentre os insetos cletrófagos que atacam o milho destacamse em importância Sitophilus zeamais Motschulsky (Coleoptera: Curculionidae) e Tribolium castaneum (Herbst) (Coleoptera: Tenebrionidae) (Elias et al., 2009).
É comum o uso de produtos químicos para a proteção dos grãos armazenados contra o ataque de pragas, como inseticidas piretroides, organofosforados e fumigantes em geral, sendo todos de alta periculosidade ecom período de carência específico. Porém, existem os métodos de controle alternativos (temperatura, radiação e som) entre os quais está o uso de pós-inertes, como a terra de diatomácea que é obtida a partir de depósitos sedimentares de sílica em águas doces e salgadas (CHANBANG et al., 2007; LORINI, 2008).

Entre as principais vantagens do uso de terra de diatomácea, pode-se citar que quando utilizada na 
dosagem recomendada não oferece riscos à saúde de quem consome os grãos ou outros seres vivos que venham a ter contato com os grãos tratados com este produto. Esse por sua vez fica livre de resíduos tóxicos e de contaminantes do meio ambiente. Por possuir ação inseticida altamente eficiente, a terra de diatomácea não compromete o controle de insetos ao longo do tempo. Este pó inerte é de fácil manuseio, não necessitando de equipamento específico quando aplicado em doses pequenas (LoRINI et al., 2001).

De acordo com o exposto, este trabalho objetivou avaliar a mortalidade eemergência deinsetos adultos das espécies $S$. zeamais e $T$. castaneum, em grãos de milho tratados com terra de diatomácea, em duas concentrações ( 1 e $\left.2 \mathrm{~kg} \cdot \mathrm{t}^{-1}\right)$, em três diferentes teores de água $(12,14$ e $16 \%)$ e em três períodos diferentes de infestação (1h, 10 e 20 dias).

\section{MATERIAL E MÉTODOS}

Utilizaram-se grãos de milho híbrido AS32 provenientes da Estação Experimental Agronômica (EEA) da Universidade Federal do Rio Grande do Sul, RS, localizada no km 47 da BR 290 em Eldorado do Sul $\left(30^{\circ} 05^{\prime} 52^{\prime \prime} \mathrm{S}, 51^{\circ} 39^{\prime} 08^{\prime \prime}\right.$ W e altitude média de $\left.46 \mathrm{~m}\right)$, durante a safra agrícola 2009/2010.

Os insetos foram criados em sala climatizada ( 25 $\pm 5^{\circ} \mathrm{C} ; 60 \pm 5 \%$ UR e fotofase de 16 horas) e mantidos em recipientes plásticos de diferentes formatos com tampa apresentando abertura vedada com tecido tipo voile para permitir as trocas gasosas.

Os indivíduos da espécie S. zeamais foram alimentados com grãos de milho híbrido AS32 cultivados na safra agrícola 2008/2009 na EEA. As trocas de dieta ocorreram em intervalos de 15 dias, com armazenagem dos grãos, que saíam do contato com os insetos, para permitir as possíveis emergências. A espécie T. castaneum foi mantida em dieta composta por farelo de trigo integral tostado, gérmen de trigo e levedo de cerveja, na mesma proporção. As fases de ovo, larva, pupa e adulto foram isoladas e mantidas em recipientes plásticos separados devido ao canibalismo.

Os grãos de milho foram secos em secador estacionário com ar natural, até teores de água de aproximadamente $13 \%$ em base úmida, exceto os grãos utilizados no experimento com terra diatomácea que apresentaram teores de água de 12, 14 e $16 \%$. Após a secagem, estes foram expurgados em sacos, envoltos por lona plástica com espessura de 200 micras, utilizou-se gás fosfina (Gastoxin), na concentração de 2 g.m ${ }^{-3}$, conforme recomendação dos fabricantes.

Aplicou-se a terra de diatomácea em $12 \mathrm{~kg}$ de grãos de milho no total dos tratamentos, sendo 2 kg para cada tratamento. A aplicação ocorreu em bandejas retangulares, com homogeneização durante 2 minutos e o armazenamento em recipientes plásticos de 2 L. Os grãos de milho apresentavam umidades de 12, 14 e $16 \%$ (b.u.) e cada uma destas foi tratada com 1.000 e $2.000 \mathrm{~g}$ de terra de diatomácea por tonelada de grãos de milho. O controle constou de grãos sem tratamentos, ou seja, livres de terra diatomácea. O produto utilizado foi da empresa Bernardo Química, com nome comercial Insecto ${ }^{\circledR}$ com $86,7 \%$ de dióxido de sílica (gentilmente doado por Protection Insumos).

Cada tratamento foi composto por cinco repetições, sendo que para cada repetição foram colocados $100 \mathrm{~g}$ de grãos de milho, em recipientes plásticos de $300 \mathrm{~mL}$ fechados com tecido tipo voile, juntamente com 10 adultos de $S$. zeamais e de $T$. castaneum, com idades variando de 20 a 50 dias, sem padronização sexual. Esses insetos foram identificados, com tinta têmpera, para evitar problemas nas verificações devido às emergências de novos insetos. As infestações ocorreram em três tempos distintos: 1 hora, 10 dias e 20 dias após a aplicação do produto nos grãos de milho. Os grãos foram armazenados por um período de 60 dias.

Avaliou-se a mortalidade aos 30 e 60 dias. Considerou-se morto o inseto que não apresentou movimentos durante dois minutos de observação. Verificou-se também a emergência dos insetos em cada recipiente, através da sua contagem.

Este experimento foi conduzido em sala climatizada nas mesmas condições da criação $\left(25 \pm 5^{\circ} \mathrm{C}\right.$; $60 \pm 5 \%$ UR e fotoperíodo de 16L:8E).

\section{RESULTADOS E DISCUSSÃO}

Em relação aos valores obtidos para emergência de S. zeamais (Tabela 1), verificaram-se que as menores médias ocorreram nos tratamentos onde foi utilizada a terra de diatomácea (TD) independente do teor de umidade dos grãos e período de infestação. Percebe-se assim a inibição de postura de ovos por parte da TD.

Com relação aos tratamentos controles, estes apresentaram médias superiores aos tratamentos com uso de TD. Nos grãos com teores de umidade igual a $12 \%$, verificaram-se menores médias independente do período de infestação, isto está relacionado ao fato que grãos com teores de umidade iguais ou inferiores a 12,5\% inibem a postura (AthiÉ; Paula, 2002).

As médias obtidas para a mortalidade de $S$. zeamais, após 30 dias de infestação, estão descritas na Tabela 2.

A maior mortalidade média de indivíduos de $S$. zeamais foi observada em grãos de milho com $12 \%$ de umidade, sendo que as maiores mortalidades ocorreram 1 hora e 10 dias após a aplicação, tanto na dose de 1.000 quanto na de 2.000 g.t $^{-1}$ de TD. 
Tabela 1 - Número médio ( \pm EP) de adultos emergidos de Sitophilus zeamais após 60 dias em grãos de milho com diferentes umidades $\left(12,14\right.$ e 16\%), tratados com terra de diatomácea (dosagens de 1.000 e 2.000 g.t $\left.t^{-1}\right)$ e controle, em função do tempo de infestação (1 hora, 10 dias e 20 dias). $\left(25 \pm 5^{\circ} \mathrm{C} ; 60 \pm 5 \%\right.$ UR e fotofase de 16 horas) ( $\left.\mathrm{n}=10\right)$.

\begin{tabular}{|c|c|c|c|c|c|}
\hline \multirow{2}{*}{ Umidade } & \multirow{2}{*}{ Tratamentos } & \multicolumn{3}{|c|}{ Período de infestação de Sitophilus zeamais após aplicação do produto } & \multirow{2}{*}{$\mathrm{CV}^{* *}$} \\
\hline & & 1 hora & 10 dias & 20 dias & \\
\hline \multirow{3}{*}{$12 \%$} & 1.000 g.t $^{-1}$ & $6,8 \mathrm{cA}( \pm 3,87)^{*}$ & $0,6 \mathrm{cA}( \pm 0,50)$ & $3,4 \mathrm{bA}( \pm 2,27)$ & 1,15 \\
\hline & 2.000 g.t $\mathrm{t}^{-2}$ & $5,6 \mathrm{cA}( \pm 3,36)$ & $1,0 \mathrm{cA}( \pm 0,59)$ & $2,0 \mathrm{bA}( \pm 1,58)$ & 1,14 \\
\hline & Controle & $34,8 \mathrm{bcA}( \pm 18,00)$ & $25,0 \mathrm{cA}( \pm 15,22)$ & $21,6 \mathrm{bA}( \pm 13,95)$ & 0,43 \\
\hline \multirow{3}{*}{$14 \%$} & 1.000 g.t $^{-1}$ & $14,4 \mathrm{cA}( \pm 7,70)$ & $0,0 \mathrm{cB}( \pm 0,00)$ & $7,2 \mathrm{bB}( \pm 4,44)$ & 1,02 \\
\hline & 2.000 g.t $\mathrm{t}^{-2}$ & $8,2 \mathrm{cA}( \pm 5,04)$ & $3,8 \mathrm{cA}( \pm 2,38)$ & 4,6 bA $( \pm 2,64)$ & 0,81 \\
\hline & Controle & 74,4 bB $( \pm 37,82)$ & 188,0 aA $( \pm 93,16)$ & $123,8 \mathrm{aAB}( \pm 73,70)$ & 0,54 \\
\hline \multirow{3}{*}{$16 \%$} & 1.000 g.t $^{-1}$ & $5,0 \mathrm{cA}( \pm 3,17)$ & $8,8 \mathrm{cA}( \pm 4,92)$ & $2,8 \mathrm{bA}( \pm 1,97)$ & 0,81 \\
\hline & 2.000 g.t. $^{-2}$ & $6,4 \mathrm{cA}( \pm 4,03)$ & $2,0 \mathrm{cA}( \pm 1,45)$ & $0,8 \mathrm{bA}( \pm 0,71)$ & 1,30 \\
\hline & Controle & $158,0 \mathrm{aA}( \pm 83,44)$ & $101,4 \mathrm{bA}( \pm 55,26)$ & $107,0 \mathrm{aA}( \pm 52,66)$ & 0,45 \\
\hline
\end{tabular}

*Médias seguidas de mesma letra minúscula na coluna ou maiúscula na linha não diferem estatisticamente pelo teste de Tukey a $1 \%$ de probabilidade.

${ }^{* *}$ C.V. $=$ coeficiente de variação a $99 \%$ de probabilidade.

Tabela 2 - Mortalidade média ( \pm EP) de adultos de Sitophilus zeamais avaliados após 30 dias, em função do tempo de infestação (1 hora, 10 dias e 20 dias), da umidade dos grãos de milho (12, 14 e 16\%) e das dosagens de terra de diatomácea (1.000 e 2.000 g.t $\left.{ }^{-1}\right)$ e controle (0). $\left(25 \pm 5^{\circ} \mathrm{C} ; 60 \pm 5 \%\right.$ UR e fotofase de 16 horas $)(n=10)$.

\begin{tabular}{|c|c|c|c|c|c|c|c|c|c|}
\hline \multirow{2}{*}{$\begin{array}{c}\text { Dose. }\left(\mathrm{g} \cdot \mathrm{t}^{-1}\right) / \\
\text { Infestação }\end{array}$} & \multicolumn{3}{|c|}{$12 \%$} & \multicolumn{3}{|c|}{$14 \%$} & \multicolumn{3}{|c|}{$16 \%$} \\
\hline & 1.000 & 2.000 & 0 & 1.000 & 2.000 & 0 & 1.000 & 2.000 & 0 \\
\hline 1 hora & $\begin{array}{c}7,6 \mathrm{aAB}^{*} \\
( \pm 1,17)\end{array}$ & $\begin{array}{l}9,4 \mathrm{aA} \\
( \pm 0,40)\end{array}$ & $\begin{array}{l}2,0 \mathrm{aC} \\
( \pm 0,84)\end{array}$ & $\begin{array}{c}4,2 \mathrm{aBC} \\
( \pm 0,73)\end{array}$ & $\begin{array}{c}4,6 \text { aBC } \\
( \pm 0,87)\end{array}$ & $\begin{array}{l}1,4 \mathrm{aC} \\
( \pm 0,40)\end{array}$ & $\begin{array}{l}5,4 \mathrm{aB} \\
( \pm 0,68)\end{array}$ & $\begin{array}{l}6,4 \mathrm{aAB} \\
( \pm 0,93)\end{array}$ & $\begin{array}{l}1,2 \mathrm{aC} \\
( \pm 0,58)\end{array}$ \\
\hline 10 dias & $\begin{array}{l}9,4 \mathrm{aA} \\
( \pm 0,40)\end{array}$ & $\begin{array}{c}10,0 \mathrm{aA} \\
( \pm 0,00)\end{array}$ & $\begin{array}{l}1,0 \mathrm{aC} \\
( \pm 0,45)\end{array}$ & $\begin{array}{c}4,4 \mathrm{aBC} \\
( \pm 0,81)\end{array}$ & $\begin{array}{c}2,8 \mathrm{aBC} \\
( \pm 1,16)\end{array}$ & $\begin{array}{l}1,4 \mathrm{aC} \\
( \pm 0,40)\end{array}$ & $\begin{array}{c}3,4 \text { aBC } \\
( \pm 1,03)\end{array}$ & $\begin{array}{c}5,2 \mathrm{aB} \\
( \pm 0,80)\end{array}$ & $\begin{array}{l}1,4 \mathrm{aC} \\
( \pm 0,40)\end{array}$ \\
\hline 20 dias & $\begin{array}{l}1,2 \mathrm{bC} \\
( \pm 0,38)\end{array}$ & $\begin{array}{c}5,8 \mathrm{bAB} \\
( \pm 0,49)\end{array}$ & $\begin{array}{c}2,6 \mathrm{aBC} \\
( \pm 0,45)\end{array}$ & $\begin{array}{c}2,8 \text { aBC } \\
( \pm 1,11)\end{array}$ & $\begin{array}{c}4,0 \mathrm{aBC} \\
( \pm 0,32)\end{array}$ & $\begin{array}{l}1,6 \mathrm{aC} \\
( \pm 0,68)\end{array}$ & $\begin{array}{c}5,2 \mathrm{aAB} \\
( \pm 0,37)\end{array}$ & $\begin{array}{l}7,4 \mathrm{aA} \\
( \pm 1,21)\end{array}$ & $\begin{array}{l}1,8 \mathrm{aC} \\
( \pm 0,58)\end{array}$ \\
\hline $\mathrm{CV}^{* *}$ & 0,63 & 0,24 & 0,73 & 0,51 & 0,49 & 0,70 & 0,37 & 0,34 & 0,74 \\
\hline
\end{tabular}

*Médias seguidas de mesma letra minúscula na coluna ou maiúscula na linha não diferem estatisticamente pelo teste de Tukey a $1 \%$ de probabilidade.

${ }^{* *}$ C.V. = coeficiente de variação a $99 \%$ de probabilidade.

Nacomparação das médias demortalidade dentro das dosagens utilizadas ao longo dos três períodos de infestação, somente ocorreram diferenças estatísticas em ambas as dosagens de TD dos grãos com $12 \%$ de umidade.

A menor mortalidade dos gorgulhos nos grãos com $12 \%$ de umidade, infestados 20 dias após a aplicação, mostra que o tempo prolongado pode ter levado a uma menor eficiência, ou seja, a TD retira água dos grãos e quando ocorre a infestação não consegue ter a mesma eficiência. Isso é observado no tratamento $14 \%$, em que a mortalidade foi inferior a $40 \%$. O aumento de eficácia em condições de produto mais seco é devido ao aumento da dessecação, que é o modo de ação da TD (Ebeling, 1971).

Massaro Junior et al. (2007) também obtiveram $100 \%$ de mortalidade de S. zeamais em grãos de milho tratados com 1000 g.t $^{-1}$ de TD (86 g.kg-1 de dióxido de sílica). Esse resultado ocorreu com sete dias de contato após a aplicação do produto. Esses autores não relataram a umidade dos grãos utilizados. Cada repetição utilizada foi composta de $100 \mathrm{~g}$ de grãos infestadas com 30 adultos.

Resultadosemelhantefoiregistrado por CANEPPELE et al. (2010) os quais obtiveram $100 \%$ de mortalidade de $S$. zeamais, 30 insetos, com 750 g.t. ${ }^{-1}$ de TD aos 21 dias em milho com $14 \%$ de umidade.

FIELDS; KorUNIC (2000), avaliando a mortalidade da espécie $S$. oryzae, 25 insetos, em grãos de trigo tratados com TD com nome comercial Protect-It, na dosagem de 300 g.t $^{-1}$, em temperatura de $25^{\circ} \mathrm{C}$, aplicados na forma de pó, em umidades de 11,8; 13,9 e $15 \%$, observaram mortalidade de 95,74 e $53 \%$, durante 5 dias de avaliação e de 97,99 e $99 \%$, respectivamente, quando avaliados por 9 dias. Segundo os autores, houve um aumento na mortalidade quando se aumentou o tempo de exposição de 5 para 9 dias, resultados semelhantes aos encontrados neste trabalho, quando se aumentou o tempo de 1 para 10 dias de exposição, na umidade de $12 \%$.

O efeito da TD $\left(\right.$ Insecto $^{\odot}$ ) foi avaliado por FIELDS; Korunic (2000) em insetos da espécie S. oryzae, 25 


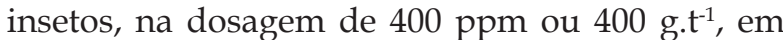
grãos de trigo, nas umidades de 12 e $14 \%$, em temperatura de $30^{\circ} \mathrm{C}$. Os autores observaram mortalidade de $96 \%$ e de $32 \%$, respectivamente para 12 e $14 \%$, e constataram que diferentes fontes de TD tiveram eficácias diferentes, mostrando que em umidades mais baixas, independentemente da fonte, existe uma maior eficiência do produto.

Quando a mortalidade de S. zeamais foi analisada aos 60 dias após a infestação, verificou-se a existência de padrão de mortalidade para ambas as dosagens de TD utilizadas nos três teores de umidade dos grãos (Tabela 3).

Em seu trabalho, VAYIAS; STEPHOU (2009) observaram que o aumento no tempo de exposição de 7 para 14 dias incrementou a mortalidade de $S$. oryzae e T. castaneum, 30 insetos de cada espécie.

O mesmo foi constatado por KLJAJIC et al. (2009), os quais registraram um aumento da mortalidade em S. oryzae e T. castaneum, 25 insetos de cada espécie, tratados com TD e expostos por 7 e 21 dias.

Neste período de análise, com exposição de 60 dias após infestação, ocorreram as maiores médias de mortalidade. Isto pode estar relacionado ao fato de que a partir do $35^{\circ}$ dia iniciou a emergência da prole dos insetos infestantes, diminuindo a quantidade de alimento disponível e forçando a locomoção dos insetos.

Quando a quantidade inicial (10 insetos) estava presente, poucos se movimentavam dificultando desta forma o contato com a TD. Nas análises, os gorgulhos geralmente foram encontrados aderidos aos grãos ou dentro deles. Esta menor mobilidade devido ao número inicial de insetos levou a uma menor mortalidade. Esses resultados corroboram com o observado por LoRINI (2008) qual constatou que a TD confere longo período de proteção à massa de grãos, sem deixar resíduos em alimentos destinados ao consumo.
Ao analisar as médias obtidas dentro de cada dose deTD, nos diferentes períodos deinfestação, percebemse diferenças estatísticas no tratamento $1.000 \mathrm{~g} . \mathrm{t}^{-1}, \mathrm{com}$ $12 \%$ deumidade, infestadosapós 20 dias com os demais períodos de infestação, já no tratamento com 2000 g.t $^{-1}$ de TD, com $14 \%$ de umidade, é o período de 10 dias após a aplicação que difere dos demais.

As outras médias tiveram comportamento igual, nos três períodos de infestação, comprovando o longo período de proteção contra insetos que a TD propicia aos grãos (LORINI 2008).

Em relação às médias obtidas para a emergência de $T$. castaneum, verificaram-se menores valores para os tratamentos com uso de TD independente do teor de umidade e momento de infestação (Tabela 4). Percebe-se assim, inibição de postura de ovos por parte da TD.

Um fator importante que deve ser levado em consideração para esses valores de emergência de $T$. castaneum é o hábito canibal desta espécie. Ocanibalismo pode ocorrer em todas as fases de desenvolvimento, ocorrendo inclusive as larvas alimentaando-se de adultos jovens (Athí́; PAULA, 2002).

Ao ser avaliada a média de mortalidade obtida com30 dias após a infestação, obteveram-se melhores resultados nos grãos com teor de umidade igual a $12 \%$, sendo que o maior valor não ultrapassou $50 \%$ de mortalidade (Tabela 5).

Ao serem comparadas as médias obtidas dentro de cada tratamento ao longo dos três períodos de infestação, somente não ocorreram diferenças estatísticas entre os tratamentos controles dos três teores de umidade utilizados.

Os dados de mortalidade obtidos neste trabalho com umidade de $12 \%$ nos grãos corroboram os obtidos por PINTO JunIOR (2008), o qual observou que, até 40 dias após a infestação, nenhuma das três doses de TD (250, 500 e 750 g.t. $^{-1}$ ) apresentou mais de $50 \%$ de mortalidade para adultos de T. castaneum.

Tabela 3 - Mortalidade média ( \pm EP) de adultos de Sitophilus zeamais avaliados após 60 dias, em função do tempo de infestação (1 hora, 10 dias e 20 dias), da umidade dos grãos de milho (12, 14 e 16\%) e das dosagens de terra de diatomácea (1.000 e 2.000 g.t $\left.t^{-1}\right)$ e controle (0). $\left(25 \pm 5^{\circ} \mathrm{C} ; 60 \pm 5 \%\right.$ UR e fotofase de 16 horas $)(n=10)$.

\begin{tabular}{|c|c|c|c|c|c|c|c|c|c|}
\hline \multirow{2}{*}{$\begin{array}{c}\text { Dose. }\left(\mathrm{g} \cdot \mathrm{t}^{-1}\right) / \\
\text { Infestação }\end{array}$} & \multicolumn{3}{|c|}{$12 \%$} & \multicolumn{3}{|c|}{$14 \%$} & \multicolumn{3}{|c|}{$16 \%$} \\
\hline & 1.000 & 2.000 & 0 & 1.000 & 2.000 & 0 & 1.000 & 2.000 & 0 \\
\hline 1 hora & $\begin{array}{c}10,0 \mathrm{aA}^{*} \\
( \pm 0,00)\end{array}$ & $\begin{array}{l}9,4 \mathrm{aA} \\
( \pm 0,40)\end{array}$ & $\begin{array}{l}2,0 \mathrm{aB} \\
( \pm 0,32)\end{array}$ & $\begin{array}{l}9,4 \mathrm{aA} \\
( \pm 0,40)\end{array}$ & $\begin{array}{c}9,0 \text { abA } \\
( \pm 0,77)\end{array}$ & $\begin{array}{l}1,8 \mathrm{aB} \\
( \pm 0,28)\end{array}$ & $\begin{array}{l}8,8 \mathrm{aA} \\
( \pm 0,58)\end{array}$ & $\begin{array}{l}9,6 \mathrm{aA} \\
( \pm 0,25)\end{array}$ & $\begin{array}{l}3,2 \mathrm{aB} \\
( \pm 1,56)\end{array}$ \\
\hline 10 dias & $\begin{array}{c}9,4 \mathrm{aAB} \\
( \pm 0,44)\end{array}$ & $\begin{array}{c}10,0 \mathrm{aA} \\
( \pm 0,00)\end{array}$ & $\begin{array}{l}2,0 \mathrm{aD} \\
( \pm 0,44)\end{array}$ & $\begin{array}{c}8,2 \mathrm{aAB} \\
( \pm 0,73)\end{array}$ & $\begin{array}{c}5,6 \\
\mathrm{bBCD} \\
( \pm 1,47)\end{array}$ & $\begin{array}{l}1,6 \mathrm{aD} \\
( \pm 0,20)\end{array}$ & $\begin{array}{c}7,0 \text { aABC } \\
( \pm 1,30)\end{array}$ & $\begin{array}{c}7,8 \mathrm{aAB} \\
( \pm 1,07)\end{array}$ & $\begin{array}{c}3,2 \mathrm{aCD} \\
( \pm 0,60)\end{array}$ \\
\hline 20 dias & $\begin{array}{l}6,8 \mathrm{bA} \\
( \pm 0,97)\end{array}$ & $\begin{array}{l}8,8 \mathrm{aA} \\
( \pm 0,73)\end{array}$ & $\begin{array}{l}3,0 \mathrm{aB} \\
( \pm 1,30)\end{array}$ & $\begin{array}{l}9,0 \mathrm{aA} \\
( \pm 0,32)\end{array}$ & $\begin{array}{l}9,8 \mathrm{aA} \\
( \pm 0,20)\end{array}$ & $\begin{array}{l}2,4 \mathrm{aB} \\
( \pm 0,40)\end{array}$ & $\begin{array}{l}7,8 \mathrm{aA} \\
( \pm 0,58)\end{array}$ & $\begin{array}{l}8,8 \mathrm{aA} \\
( \pm 0,49)\end{array}$ & $\begin{array}{l}3,0 \mathrm{aB} \\
( \pm 0,40)\end{array}$ \\
\hline $\mathrm{CV}^{* *}$ & 0,21 & 0,12 & 0,68 & 0,13 & 0,33 & 0,35 & 0,25 & 0,18 & 0,64 \\
\hline
\end{tabular}

*Médias seguidas de mesma letra minúscula na coluna ou maiúscula na linha não diferem estatisticamente pelo teste de Tukey a $1 \%$ de probabilidade.

${ }^{* *}$ C.V. $=$ coeficiente de variação a $99 \%$ de probabilidade. 
Tabela 4 - Número médio ( \pm EP) de adultos emergidos de Tribolium castaneum após 60 dias em grãos de milho com diferentes umidades $\left(12,14\right.$ e $16 \%$ ), tratados com terra de diatomácea (dosagens de 1.000 e 2.000 g.t $^{-1}$ ) e controle (0), em função do tempo de infestação (1 hora, 10 dias e 20 dias). ( $25 \pm 5^{\circ} \mathrm{C} ; 60 \pm 5 \%$ UR e fotofase de 16 horas) $(\mathrm{n}=10)$.

\begin{tabular}{|c|c|c|c|c|c|}
\hline \multirow{2}{*}{ Umidade } & \multirow{2}{*}{ Tratamentos } & \multicolumn{3}{|c|}{ Período de infestação de Sitophilus zeamais após aplicação do produto } & \multirow{2}{*}{$\mathrm{CV}^{* *}$} \\
\hline & & 1 hora & 10 dias & 20 dias & \\
\hline \multirow{3}{*}{$12 \%$} & 1000 g.t $t^{-1}$ & $0,0 \mathrm{bA}( \pm 0,00)$ & $0,0 \mathrm{aA}( \pm 0,00)$ & $0,4 \mathrm{bA}( \pm 0,32)$ & 2,55 \\
\hline & 2000 g.t $t^{-2}$ & $0,0 \mathrm{bA}( \pm 0,00)$ & $0,0 \mathrm{aA}( \pm 0,00)$ & $0,2 \mathrm{bA}( \pm 0,22)$ & 3,74 \\
\hline & Controle & $0,0 \mathrm{bB}( \pm 0,00)$ & $0,0 \mathrm{aB}( \pm 0,00)$ & $2,0 \mathrm{aA}( \pm 5,71)$ & 1,61 \\
\hline \multirow{3}{*}{$14 \%$} & 1000 g.t $\mathrm{t}^{-1}$ & $0,0 \mathrm{bA}( \pm 0,00)$ & 0,0 aA $( \pm 0,00)$ & $0,0 \mathrm{bA}( \pm 0,00)$ & 0,00 \\
\hline & 2000 g.t $\mathrm{t}^{-2}$ & $0,0 \mathrm{bA}( \pm 0,00)$ & 0,0 aA $( \pm 0,00)$ & $0,0 \mathrm{bA}( \pm 0,00)$ & 0,00 \\
\hline & Controle & $4,8 \mathrm{aA}( \pm 3,67)$ & $0,0 \mathrm{aB}( \pm 0,00)$ & $0,6 \mathrm{bB}( \pm 0,67)$ & 2,18 \\
\hline \multirow{3}{*}{$16 \%$} & 1000 g.t. & $0,0 \mathrm{bA}( \pm 0,00)$ & 0,0 aA $( \pm 0,00)$ & $0,0 \mathrm{bA}( \pm 0,00)$ & 0,00 \\
\hline & 2000 g.t $t^{-2}$ & $0,0 \mathrm{bA}( \pm 0,00)$ & 0,0 aA $( \pm 0,00)$ & $0,0 \mathrm{bA}( \pm 0,00)$ & 0,00 \\
\hline & Controle & $0,0 \mathrm{bA}( \pm 0,00)$ & 0,0 aA $( \pm 0,00)$ & $0,2 \mathrm{bA}( \pm 0,22)$ & 2,71 \\
\hline
\end{tabular}

*Médias seguidas de mesma letra minúscula na coluna ou maiúscula na linha não diferem estatisticamente pelo teste de Tukey a $1 \%$ de probabilidade.

${ }^{* *}$ C.V. $=$ coeficiente de variação a $99 \%$ de probabilidade.

Tabela 5 - Mortalidade média ( \pm EP) de adultos de Tribolium castaneum avaliados após 30 dias, em função do tempo de infestação (1 hora, 10 dias e 20 dias), da umidade dos grãos de milho (12, 14 e 16\%) e das dosagens de terra de diatomácea (1.000 e 2.000 g.t $\left.t^{-1}\right)$ e controle $(0)\left(25 \pm 5^{\circ} \mathrm{C} ; 60 \pm 5 \%\right.$ UR e fotofase de 16 horas $)(n=10)$.

\begin{tabular}{|c|c|c|c|c|c|c|c|c|c|}
\hline \multirow{2}{*}{$\begin{array}{l}\text { Dose }\left(\mathrm{g} \cdot \mathrm{t}^{-1}\right) / \\
\text { Exposição }\end{array}$} & \multicolumn{3}{|c|}{$12 \%$} & \multicolumn{3}{|c|}{$14 \%$} & \multicolumn{3}{|c|}{$16 \%$} \\
\hline & 1.000 & 2.000 & 0 & 1.000 & 2.000 & 0 & 1.000 & 2.000 & 0 \\
\hline 1 hora & $\begin{array}{c}2,0 \mathrm{abA}^{*} \\
( \pm 0,83)\end{array}$ & $\begin{array}{c}1,2 \mathrm{bAB} \\
( \pm 0,58)\end{array}$ & $\begin{array}{l}0,6 \mathrm{aB} \\
( \pm 0,25)\end{array}$ & $\begin{array}{l}2,2 \mathrm{aA} \\
( \pm 0,79)\end{array}$ & $\begin{array}{l}2,2 \mathrm{aA} \\
( \pm 1,20)\end{array}$ & $\begin{array}{l}0,4 \mathrm{aB} \\
( \pm 0,25)\end{array}$ & $\begin{array}{c}1,2 \mathrm{aAB} \\
( \pm 0,37)\end{array}$ & $\begin{array}{c}1,6 \mathrm{aAB} \\
( \pm 0,68)\end{array}$ & $\begin{array}{r}1,2 \mathrm{aAB} \\
( \pm 0,73)\end{array}$ \\
\hline 10 dias & $\begin{array}{c}4,4 \mathrm{aAB} \\
( \pm 1,69)\end{array}$ & $\begin{array}{l}5,0 \text { aA } \\
( \pm 1,10)\end{array}$ & $\begin{array}{c}1,2 \mathrm{aBC} \\
( \pm 0,68)\end{array}$ & $\begin{array}{l}0,0 \mathrm{bC} \\
( \pm 0,00)\end{array}$ & $\begin{array}{l}0,6 \mathrm{bC} \\
( \pm 0,49)\end{array}$ & $\begin{array}{l}0,0 \mathrm{aC} \\
( \pm 0,00)\end{array}$ & $\begin{array}{l}0,0 \mathrm{bC} \\
( \pm 0,00)\end{array}$ & $\begin{array}{c}0,6 \mathrm{aC} \\
( \pm 0,40)\end{array}$ & $\begin{array}{l}0,6 \mathrm{aC} \\
( \pm 0,60)\end{array}$ \\
\hline 20 dias & $\begin{array}{l}0,0 \mathrm{bA} \\
( \pm 0,00) \\
\end{array}$ & $\begin{array}{l}1,0 \mathrm{bA} \\
( \pm 0,55) \\
\end{array}$ & $\begin{array}{l}0,8 \mathrm{aA} \\
( \pm 0,80) \\
\end{array}$ & $\begin{array}{l}0,2 \mathrm{bA} \\
( \pm 0,20) \\
\end{array}$ & $\begin{array}{l}0,0 \mathrm{bA} \\
( \pm 0,00) \\
\end{array}$ & $\begin{array}{l}0,0 \mathrm{aA} \\
( \pm 0,00) \\
\end{array}$ & $\begin{array}{c}0,4 \text { abA } \\
( \pm 0,25)\end{array}$ & $\begin{array}{l}0,2 \mathrm{bA} \\
( \pm 0,20)\end{array}$ & $\begin{array}{l}0,4 \mathrm{aA} \\
( \pm 0,40) \\
\end{array}$ \\
\hline $\mathrm{CV}^{* *}$ & 1,32 & 1,01 & 1,51 & 1,78 & 1,81 & 2,55 & 1,35 & 1,38 & 1,67 \\
\hline
\end{tabular}

*Médias seguidas de mesma letra minúscula na coluna ou maiúscula na linha não diferem estatisticamente pelo teste de Tukey a $1 \%$ de probabilidade.

${ }^{* *}$ C.V. = coeficiente de variação a $99 \%$ de probabilidade.

A baixa mortalidade de T. castaneum está relacionada ao fato deste inseto permanecer no interior dos grãos danificados, ficando menos expostos à TD. No presente estudo não foram encontrados insetos mortos no interior de grãos e a presença deles foi observada no interior do mesmo grão durante diversas avaliações.

Outro fato importanteécom relação à mobilidade, visto que insetos da espécie Tribolium se movimentam pouco no interior da massa de grãos, dificultando o contato com a TD. Rigaux et al. (2001) verificaram que adultos de $T$. castaneum menos ágeis foram mais tolerantes à TD (Protect-It). Assim, a baixa mortalidade pode levar à resistência, considerada resistência comportamental e não resistência fisiológica, uma vez que se baseia, principalmente, na diminuição do contato com as partículas do produto (VAYIAS et al., 2008).

Resultados de baixa mortalidade e resistência de Tribolium também foram relatados por FIELDS; Korunic (2000). Conforme esses autores, embora existam classificações diferentes entre os estudos com resistência a TD, os rankings são globais indo dos insetos mais sensíveis aos menos sensíveis, conforme segue: Cryptolestes spp., Oryzaephilus spp., Sitophilus spp., R. dominica e Tribolium spp.

Poucos estudos têm abordado a existência de diferença na susceptibilidade entre as espécies. Porém os mais resistentes são insetos das espécies Tribolium. FIELDS; KORUNIC (2000), em bioensaios com T. castaneum, 25 insetos, em grãos de trigo tratados com 600 g.t-1 de TD, com nome comercial Insecto@, em temperatura de $30^{\circ} \mathrm{C}$, em umidades de 12 e $14 \%$, observaram uma mortalidade de $66 \%$ e de $30 \%$, respectivamente, para as duas umidades estudadas.

Outros fatores que podem explicar as diferenças na suscetibilidade de insetos à terra de diatomácea relacionam-se ao tamanho do organismo (volume e razão deárea de superfície), diferenças quantitativas ou qualitativas em lipídios cuticulares, reação comportamental à TD e níveis de dessecação (KORUNIC, 1997; FIELDS; KORUNIC, 2000). 
EBELING (1971) considerou que a resistência genética à TD é improvável devido ao modo de ação física do produto. No entanto, Korunic (1998) e Korunic; ORMESHER (1999) descobriram susceptibilidade reduzida (1,3 - 2,2 vezes), de T. castaneum, C. ferrugineus e $R$. dominica adultos expostos a TD por 5-7 gerações, em comparação com linhagens de laboratório não selecionadas. Isto significa que os insetos podem ser capazes de desenvolver mecanismos de resistência fisiológica ou comportamental ao produto.

Já consta na literatura a informação sobre determinada população de $T$. castaneum, que nunca tinha estado em contacto com TD, foi naturalmente tolerante à formulação do produto com nome comercial Protect-It (Rigaux et al., 2001). Portanto, encontrar uma fórmula eficaz contra uma população de uma espécie não permite a extrapolação dos resultados para todas as populações das espécies examinadas.

ARNAUD et al. (2005) observaram a mortalidade de adultos de sete populações distintas de $T$. castaneum, 50 insetos, a quatro diferentes produtos comerciais à base de TD, aplicados em grãos de trigo, com umidade de 13-14\%, nas concentrações de 100, 200, 400, 600, 800 e 1.000 ppm (mg de TD por kg de grão). Após 21 dias os insetos foram contados, sendo observado que, no produto Insecto ${ }^{\odot}$, a mortalidade foi de $100 \%$ para três das sete populações tratadas com 400 ppm, a menor média foi de $12 \%$ para uma população. Já com 600 ppm apenas duas populações não foram controladas completamente, 23 e $96 \%$ de mortalidade. Ao usarem 1.000 ppm, os autores obtiveram $100 \%$ de mortalidade em seis populações e $97 \%$ em uma população, mostrando mais uma vez que dependendo da origem da população teremos diferente eficiência no controle (Rigaux et al., 2001).

O uso da TD em T. castaneum não é maior devido à necessidade de grandes concentrações, variando de 1000 - 3500 ppm (1000 - 3500 g.t $\left.^{-1}\right)$ para se alcançar uma boa eficiência, o que reduz significativamente a densidade e fluidez de grãos, e cria resíduos de poeira visíveis, aumentando os custos de aplicação (GolOB, 1997).

Outro fator importante que pode estar relacionada à baixa mortalidade observada nesta especie, no presente trabalho, está relacionado com o tipo de grão utilizado. Foi observado que TD foi mais eficiente quando aplicada sobre trigo do que quando aplicada em outros grãos, tais como, arroz polido, milho, aveia e cevada (VAYIAS; STEPHOU, 2009). Estes autores, estudando a mortalidade de insetos da espécie S. oryzae e T. castaneum em grãos de cevada, trigo, arroz e milho, após tratamento por 14 dias com um produto à base de TD, observaram menor mortalidade das duas espécies quando o produto foi aplicado em milho, do que nos outros grãos.

De acordo com ARNAUD et al. (2005), provavelmente a dosagem de TD terá de ser aumentada em determinados grãos para obter um controle semelhante ao do trigo. Athanassiou et al. (2003) relataram que no milho existem grandes espaços entre os grãos, que permitem que os insetos ao andar através desses espaços evitam entrar em contato com as áreas onde a quantidade de TD é mais alta. Segundo os mesmos autores, a porosidade em grãos de milho é maior que em outros grãos.

Além disso, variações nas características físicoquímicas entre os grãos poderia explicar a diferente eficiência de TD. Por exemplo, a quantidade de produto que é retida na superfície de grãos é diferente entre os grãos, e poderia levar a diferenças na eficiência do produto (VAYIAS; STEPHOU, 2009).

Esses resultados destacam a necessidade de estudar a suscetibilidade de diversas populações de diferentes espécies com uso de TD, a fim de desenvolver formulações eficazes contra a maioria das espécies de insetos e das populações.

Ao verificar a presença de $T$. castaneum 60 dias após a infestação, observaram-se que os melhores resultados foram obtidos nos grãos com teor de umidade igual a $12 \%$ (Tabela 6).

Tabela 6 - Mortalidade média ( \pm EP) de adultos de Tribolium castaneum avaliados após 60 dias, em função do tempo de infestação (1 hora, 10 dias e 20 dias), da umidade dos grãos de milho (12, 14 e $16 \%)$ e das dosagens de terra de diatomácea (1.000 e 2.000 g.t $\left.\mathrm{t}^{-1}\right)$ e controle (0). $\left(25 \pm 5^{\circ} \mathrm{C} ; 60 \pm 5 \%\right.$ UR e fotofase de 16 horas) $(\mathrm{n}=10)$.

\begin{tabular}{|c|c|c|c|c|c|c|c|c|c|}
\hline \multirow{2}{*}{$\begin{array}{c}\text { Dose. }\left(\mathrm{g} \cdot \mathrm{t}^{-1}\right) / \\
\text { Infestação }\end{array}$} & \multicolumn{3}{|c|}{$12 \%$} & \multicolumn{3}{|c|}{$14 \%$} & \multicolumn{3}{|c|}{$16 \%$} \\
\hline & 1.000 & 2.000 & 0 & 1.000 & 2.000 & 0 & 1.000 & 2.000 & 0 \\
\hline 1 hora & $\begin{array}{c}5,2 \mathrm{abA}^{*} \\
( \pm 1,02)\end{array}$ & $\begin{array}{c}4,2 \text { aAB } \\
( \pm 1,24)\end{array}$ & $\begin{array}{c}2,4 \mathrm{aAB} \\
( \pm 0,40)\end{array}$ & $\begin{array}{c}2,8 \mathrm{aAB} \\
( \pm 1,16)\end{array}$ & $\begin{array}{c}3,6 \mathrm{aAB} \\
( \pm 0,93)\end{array}$ & $\begin{array}{l}0,4 \mathrm{aB} \\
( \pm 0,25)\end{array}$ & $\begin{array}{c}2,8 \mathrm{aAB} \\
( \pm 0,85)\end{array}$ & $\begin{array}{c}2,8 \text { aAB } \\
( \pm 1,11)\end{array}$ & $\begin{array}{r}1,8 \mathrm{aAB} \\
( \pm 0,73)\end{array}$ \\
\hline 10 dias & $\begin{array}{l}7,4 \mathrm{aA} \\
( \pm 0,87)\end{array}$ & $\begin{array}{l}6,8 \mathrm{aA} \\
( \pm 1,14)\end{array}$ & $\begin{array}{l}2,0 \mathrm{aB} \\
( \pm 1,26)\end{array}$ & $\begin{array}{l}0,0 \mathrm{bB} \\
( \pm 0,00)\end{array}$ & $\begin{array}{c}0,6 \mathrm{bB} \\
( \pm 0,25)\end{array}$ & $\begin{array}{l}2,2 \mathrm{aB} \\
( \pm 1,36)\end{array}$ & $\begin{array}{l}0,6 \mathrm{aB} \\
( \pm 0,40)\end{array}$ & $\begin{array}{l}1,4 \mathrm{aB} \\
( \pm 0,51)\end{array}$ & $\begin{array}{l}1,4 \mathrm{aB} \\
( \pm 0,60)\end{array}$ \\
\hline 20 dias & $\begin{array}{l}4,0 \mathrm{bA} \\
( \pm 0,55)\end{array}$ & $\begin{array}{l}3,8 \text { aA } \\
( \pm 0,66)\end{array}$ & $\begin{array}{l}1,0 \mathrm{aB} \\
( \pm 0,71)\end{array}$ & $\begin{array}{l}0,4 \mathrm{bB} \\
( \pm 0,25)\end{array}$ & $\begin{array}{l}0,2 \mathrm{bB} \\
( \pm 0,20)\end{array}$ & $\begin{array}{l}0,4 \mathrm{aB} \\
( \pm 0,40)\end{array}$ & $\begin{array}{l}1,0 \mathrm{aB} \\
( \pm 0,55)\end{array}$ & $\begin{array}{l}1,5 \mathrm{aB} \\
( \pm 0,58)\end{array}$ & $\begin{array}{l}0,4 \mathrm{aB} \\
( \pm 0,40)\end{array}$ \\
\hline $\mathrm{CV}^{* *}$ & 0,40 & 0,49 & 1,04 & 1,73 & 1,29 & 1,86 & 1,05 & 0,96 & 1,11 \\
\hline
\end{tabular}

*Médias seguidas de mesma letra minúscula na coluna ou maiúscula na linha não diferem estatisticamente pelo teste de Tukey a $1 \%$ de probabilidade.

${ }^{* *}$ C.V. = coeficiente de variação a $99 \%$ de probabilidade. 
As baixas médias de mortalidade estão relacionadas ao fato de $T$. castaneum permanecer no interior de grãos já avariados, quebrados ou carunchados principalmente, e ali se alimentarem continuamente, evitando-se assim a locomoção e consequentemente o contato com a TD.

Resultados esses que corroboram os obtidos por FieldS; KorUNIC (2000), os quais obtiveram sempre maior mortalidade de T. castaneum, 25 insetos, nas menores umidades estudadas em grãos de trigo tratados com Insecto ${ }^{\odot}$.

Ao comparar as médias de mortalidade em cada dose de TD avaliada, na mesma umidade, nos três períodos de infestação estudados, podemos observar que ocorreram diferenças estatísticas do período 20 dias com o de 10 dias na umidade de $12 \%$ com 1.000 g.t ${ }^{-1}$ de TD. Já com 14\% de umidade, em ambos os tratamentos, ocorreram diferenças entre 1 hora de infestação com os demais períodos.

De acordo com os resultados, quanto maior o tempo que o grão permanecer com o produto sem a presença dos insetos, menor é a sua eficiência. Isto ocorre, provavelmente, porque a TD irá retirar água dos grãos perdendo o seu poder de ação contra o inseto.

\section{CONCLUSÕES}

A mortalidade de $S$. zeamais e $T$. castaneum aumenta nas menores umidades dos grãos, com o incremento no tempo de exposição dos insetos e a dose de aplicação não influencia.

A espécie T. castaneum apresentou maior nível de tolerância às doses de Terra de Diatomácea utilizadas.

Grãos de milho sem Terra de Diatomácea são danificados por S. zeamais e T. castaneum e em dois meses podem passar da classificação tipo 1 para a classificação abaixo do nível padrão de comercialização.

\section{REFERÊNCIAS}

ARNAUD, L.; LAN, H.T.T.; BROSTAUX, Y.; HAUBRUGE, E. Efficacy of diatomaceous earth formulations admixed with grain against populations of Tribolium castaneum. Journal of Stored Products Research, v.41, p.121-130, 2005.

ATHANASSIOU, C.G.; KAVALLIERATOS, N.G.; TSAGANOU, F.C.; VAYIAS, B.J.; DIMIZAS, C.B.; BUCHELOS, C.T.H. Effect of grain type on the insecticidal efficacy of diatomaceous earth against Sitophilus oryzae (L.) (Coleoptera: Curculionidae). Crop Protection, v.22, p.1141-1147, 2003.

ATHIÉ, I.; PAULA, C. de Insetos de grãos armazenados aspectos biológicos e identificação. São Paulo: Varela, 2002. 244p.
CANEPPELE, M.A.B.; ANDRADE, P.J.; SANTAELLA, A.G. Diferentes dosagens de pó inerte e temperaturas em milho armazenado para controle de gorgulho-do-milho. Scientia Agraria, v.11, p.343-347, 2010.

CHANBANG, Y.; ARTHUR, F.H.; WILDE, G.E.; THRONE, J. E. Diatomaceous earth plus methoprene for control of the lesser grain borer, Rhyzopertha dominica (F.) (Coleoptera: Bostrichidae) in rough rice. Journal of Stored Products Research, v.43, p.396-401, 2007.

EBELING, W. Sorptive dusts for pest control. Annual Review of Entomology, v.16, p.123-158, 1971.

ELIAS, M.C.; LORINI, I.; MALLAMANN, C.A.; DILKIN, P.; OLIVEIRA, M.; MALLMANN, A.O. Manejo integrado no controle de pragas de grãos e derivados. In: ELIAS, M.C.; OLIVEIRA, M. (Ed.). Aspectos tecnológicos e legais na formação de auditores técnicos do Sistema $\mathrm{Na}$ cional de Certificação de Unidades Armazenadoras. Pelotas: Super Copías Santa Cruz, 2009. 430p. Cap. 5.

FIELDS, P.G.; KORUNIC, Z. The effect of grain moisture content and temperatures on the efficacy of diatomaceous earths from different geographical locations against stored-products beetles. Journal of Stored Products Research, v.36, p.1-13, 2000.

GOLOB, P. Current status and future perspectives for inert dusts for control of stored product insects. Journal of Stored Products Research, v.33, p.69-79, 1997.

KLJAJIC, P.; ANDRIC, G.; ADAMOVIC, M.; BODROZASOLAROV, M.; MARKOVIC, M.; PERIC, I. Laboratory assessment of insecticidal effectiveness of natural zeolite and diatomaceous earth formulations against three stored-product beetle pests. Journal of Stored Products Research, v.45, p.1-6, 2009.

KORUNIC, Z. Rapid assessment of the insecticidal value of diatomaceous earths without conducting bioassays. Journal of Stored Products Research, v.33, p.219-229, 1997.

KORUNIC, Z. Diatomaceous earts, a group of natural insecticides. Journal of Stored Products Research, v.34, p.97-97, 1998.

KORUNIC, Z.; ORMESHER, P. Evaluation and standardised testing of diatomaceous earth. Ed. ZUXUN, J.; QUAN, L.; YONGSHENG, L.; XIANCHANG, T.; LIANGHUA, G. In: INTERNATIONAL WORKING CONFERENCE ON STORED-PRODUCT PROTECTION, 7., 1998, Beijing. Proceedings. Beting: Sichuan Publishing House of Science and Technology, 1999. p.738-744.

LORINI, I. Manejo integrado de pragas de grãos de cereais armazenados. Passo Fundo: Embrapa Trigo, 2008. 96p.

LORINI, I.; FERREIRA FILHO, A.; BARBIERI, I.; DEMAMAN, N. A.; MARTINS, R.R.; DALBELLO, O. Terra 
de diatomáceas como alternativa no controle de pragas de milho armazenado em propriedade familiar. Agroecologia e Desenvolvimento Rural Sustentável, v.2, p.32-36, 2001.

LORINI, I.; MIIKE, L.H.; SCUSSEL, V.M. Armazenagem de grãos. Campinas, SP: Instituto Biogeneziz, 2002. v.1, $1000 \mathrm{p}$.

MASSARO JUNIOR, A.L.; MOURÃO JUNIOR, M.; PAIVA, W.R.S.C.; BARRETO, H.C.S. Eficiência da terra de diatomácea no controle de Sitophilus zeamais em milho armazenado. Revista Acadêmica: Ciências Agrárias e Ambientais, v.5, p.27-32, 2007.

RIGAUX, M.; HAUBRUGE, E.; FIELDS, P. G. Mechanisms for tolerance to diatomaceous earth between strains of Tribolium castaneum. Entomologia Experimentalis et Applicata, v.101, p.33-39, 2001.
PINTO JUNIOR, A.R. Eficiência de terra de diatomácea no controle de algumas pragas de milho armazenado a granel. Revista da Faculdade de Zootecnia Veterinária e Agronomia, v.15, p.61-70, 2008.

VAYIAS, B.J.; STEPHOU, V.K. Factors affecting the insecticidal efficacy of an enhanced diatomaceous earth formulation against three stored-product insect species. Journal of Stored Products Research, v.45, p.226-231, 2009.

VAYIAS, B.J.; ATHANASSIOU, C.G.; BUCHELOS, C.T. Evaluation of resistance development by Tribolium confusum Du Val (Coleoptera: Tenebrionidae) to diatomaceous earth under laboratory selection. Journal of Stored Products Research, v.44, p.162-168, 2008.

Recebido em 28/4/11

Aceito em 8/4/13 